\title{
Factors influencing exercise participation by older adults requiring chronic hemodialysis: A qualitative study
}

Kontos, P., Miller, K.L., Brooks, D., Jassal, S.V., Spanjevic, L.,
Devins, G.M., De Souza, M.J., Heck, C., Laprade, J., Naglie, G.

Version Post-Print/Accepted Manuscript

Citation Kontos, P., Miller, K.L., Brooks, D., Jassal, S.V., Spanjevic, L., Devins, (published version) G.M., De Souza, M.J., Heck, C., Laprade, J., Naglie, G. Factors influencing exercise participation by older adults requiring chronic hemodialysis: A qualitative study. International Urology and Nephrology 2007; 39:1303-1311.

Publisher's Statement The final publication is available at Springer via http://dx.doi.org/10.1007/s11255-007-9265-z. accepted manuscript) because you cannot access the published version, then cite the TSpace version in addition to the published version using the permanent URI (handle) found on the record page. 


\section{Factors Influencing Exercise Participation by Older Adults Requiring Chronic Hemodialysis: A Qualitative Study}

Pia C. Kontos, PhD, MA ${ }^{1,2}$, Karen-Lee Miller, MSW, $\mathrm{MA}^{2}$, Dina Brooks, PhD, MSc ${ }^{1,3}$, Sarbjit Vanita Jassal, MD, MSc ${ }^{4,5}$, Lily Spanjevic, RN, MN ${ }^{1}$, Gerald Michael Devins, PhD, C.Psych ${ }^{6}$, Mary Jane De Souza, PhD, FACSM ${ }^{7}$, Carol Heck, PhD, $\mathrm{MSc}^{8}$, Judith Laprade, PhD, MSc ${ }^{3}$, Gary Naglie, MD, FRCPC ${ }^{1,5,9,10}$

${ }^{1}$ Toronto Rehabilitation Institute, ${ }^{2}$ Department of Public Health Sciences, University of Toronto ${ }^{3}$ Deparment of Physical Therapy, University of Toronto, ${ }^{4}$ Division of Nephrology, University Health Network, ${ }^{5}$ Department of Health Policy, Management and Evaluation, University of Toronto, ${ }^{6}$ Department of Behavioural Science, University Health Network and Department of Psychiatry, University of Toronto, ${ }^{7}$ Faculty of Physical Education and Health, University of Toronto, ${ }^{8}$ Research, University Health Network, ${ }^{9}$ Department of Medicine, University of Toronto, ${ }^{10}$ Division of General Internal Medicine and Toronto General Research Institute, University Health Network.

Corresponding author:

Pia C. Kontos

Research Scientist

Toronto Rehabilitation Institute 11035-550 University Ave.

Toronto, ON M5G 2A2

Canada

FAX: (416) 597-7105

Email: kontos.pia@torontorehab.on.ca

Running head: Factors influencing exercise in older ESRD patients 


\section{ABSTRACT}

Despite the recognized health and psychosocial benefits to exercise in older adults with end-stage renal disease (ESRD), exercise participation remains poor. Previous research has attributed low levels of exercise to patient-related factors such as lack of motivation and fear of adverse consequences. This qualitative study involving focus group discussions with hemodialysis patients, nephrology nurses and family care providers explored specific motivators and barriers to exercise participation in older adults requiring hemodialysis. Nurse participants were chosen for the health care provider focus groups because their prolonged and sustained contact with hemodialysis patients during the dialysis treatment sessions positions them well to encourage and promote exercise. Motivators to exercise included patient aspirations to exercise and their experiences of improvements from exercising, as well as the formal incorporation of exercise into the overall dialysis treatment plan. Barriers to exercise included nurses' lack of encouragement to exercise, transportation issues, and the use of exercise equipment that precludes participation by patients who recline during dialysis, and inhibits exercise encouragement by nurses due to concerns of equipment-related injury. These findings support the need for a broader recognition of the systemic factors that may impede exercise participation by older adults requiring hemodialysis. A shift is required in the culture of ESRD treatment programs towards a wellness perspective that includes expectations of exercise encouragement by the health care team, and participation by patients.

Key words: hemodialysis, end stage renal disease, exercise, older adults, focus groups 


\section{INTRODUCTION}

As a consequence of end-stage renal disease (ESRD) and its sequelae, patients often demonstrate reduced physical capacity and functional impairment $[1,2]$. These limitations are greater in older dialysis patients [3], the most rapidly growing segment of the dialysis population [4], and have been shown to compromise their rehabilitation potential [5]. Studies have demonstrated that many of the sequelae of ESRD are caused or are influenced by deconditioning [6-8], and that exercise during dialysis or on non-dialysis days can significantly improve many of these outcomes, even in older patients [6-9]. In light of these benefits, it is recommended that exercise be incorporated into standard ESRD care [10]. Nonetheless, exercise remains extremely low in dialysis patients $[6,11]$, which has generally been attributed to patient related factors such as lack of motivation $[12,13]$. The absence of exercise counseling practices among nephrologists [14] and other dialysis staff [15] may also contribute to patients' low levels of exercise.

The successful implementation of an exercise program for older hemodialysis patients is largely dependent upon an understanding of the factors influencing exercise participation. However, research has rarely explored the barriers and facilitators most salient to older hemodialysis patients [13] and their care providers [15]. The objective of this study was to expand such knowledge in order to increase exercise participation in this patient population.

\section{METHODS}

This study was conducted at three outpatient hemodialysis units of two large urban hospitals in Toronto, Canada. Non-probability sampling [16] was used to select key hemodialysis exercise informants. Patient eligibility included: being 65 years of age or older; having grade 6 or higher English fluency; and being on hemodialysis for at least six months. Staff were eligible if they were registered as a nurse or practical nurse and had a minimum of one year dialysis 
experience. Nurses were chosen because they play an integral role in providing dialysis care and are well positioned to promote exercise given their prolonged and sustained contact with patients during dialysis sessions. Family care providers were eligible if they resided with a hemodialysis patient and provided emotional support and/or assistance with activities of daily living. All participants were required to speak English and provide informed consent. Ethics approval was obtained by the ethics review board of each study hospital.

Exercise-related beliefs and experiences of patients, nurses, and family care providers were explored using focus group discussions, as this method facilitates exploratory research in areas in which little is known [17]. Nine audio-taped, semi-structured focus groups (see Appendix for focus group guides) lasting approximately one hour were facilitated by the first author (PK). At one hospital, focus groups were conducted with family care providers (one group, $n=4$ ), patients (two groups, $n=7$ and $n=6$ ), and nurses (three groups, $n=8, n=5$ and $n=5$ ). At the other, one focus group with each participant category was conducted (patients, $n=4$; nurses, $n=6$; family care providers, $n=4$ ). Data collection ceased at this point since no new information was emerging (i.e. saturation had been reached) [18].

All focus groups were transcribed and analyzed according to standard qualitative analysis techniques [19]. Qualitative data analysis begins with an inductive descriptive process of sorting and defining the data [20]. Two research team members divided the text of the transcripts into segments of text and attached descriptive codes of analysis to these segments [21]. The descriptive codes were then grouped into the broad topic-oriented categories of micro-and macro-level barriers and facilitators and all text segments belonging to the same category were compared [21]. To ensure that the data were coded in a consistent manner, "inter-coder" agreement [22] was established by cross checking independently coded transcripts. When 
inconsistency was found, coding schemes were discussed and further refined until agreement was achieved. The credibility of the interpretations was further insured by the comparison of coded and raw data by a senior team member (GN).

\section{RESULTS}

Characteristics of the study participants are described in the Table. Six themes related to exercise were identified: four barriers (ESRD, its sequelae, and other co-morbidities; transportation; time; and equipment) and two facilitators (sources of motivation; and the formal incorporation of exercise into the treatment plan).

\section{Barriers to Exercise}

ESRD, its Sequelae, and Other Co-Morbidities

Many patients and family care providers discussed the challenge of engaging in exercise because of ESRD and its sequelae. Fatigue was the most prominent symptom discussed. As one patient commented:

You need to have some time to recuperate from dialysis. As you know it's exhausting, and it takes a while until you get over it, a few hours. I mean, overnight sleep is fine. But you find yourself in the morning exhausted, and still you want to sleep again. I don't know about other people but that is how I feel.

In reference to his wife who receives dialysis treatment, a family care provider similarly commented: "Dialysis saps your energy. The fatigue, oh the fatigue." Comorbidities of various kinds were experienced by all patient participants and were identified as barriers to exercise as exemplified in the following statement:

I feel somewhat exhausted after a bit of exertion, you know. Of course I have a bit of a breathing problem, bronchitis, that goes with the lung disease, taking puffers and so on. I also had a double bypass. I had a lot of things happening, that may have something to do with why I don't exercise. Having kidney disease, heart disease, lung disease and the rest of it and being the age I am, I'm 76 years old now, that doesn't really put you in the frame of mind to start any marathon. 
Another patient linked her co-morbidities and related pain to feelings of depression, and identified her depression as a barrier to exercise participation:

And we all have osteoporosis at some level, and arthritis. And the pain and the discomfort... ruins your will to continue. You say to yourself, "it's always some other thing" and "what good is it doing to me?" and you can get very depressed about it. And I think that takes away the will to do any exercise.

\section{Transportation}

In Toronto, Canada, disabled individuals are provided access to a publicly-funded, feefor-service accessible transit service called Wheel-Trans. Since pick-up times depend on the distance traveled and whether rides are shared with other passengers, dialysis patients must sometimes agree to pick up times that are hours in advance of treatment and wait long periods of time. As one nurse indicated, "They will come at seven-thirty in the morning for dialysis 'till twelve o'clock, and they will tell you at six o'clock they were waiting outside for their WheelTrans, so by the time they get in they are really tired". Wheel-Trans drivers are instructed to wait no more than five minutes past the scheduled pickup time, and three missed rides in a row results in the temporary suspension of riding privileges. Consequently, patients' fear of the penalties resulting from missed or late rides was identified by nurses as a factor impeding exercise practice. As one nurse commented, "One of the barriers with...exercise...[is] the Wheel-Trans because people are fearful of losing their Wheel-Trans. If they lose it, it's a big deal." So great were patient worries over penalties that nurses commented that it was commonplace for patients to request shortened dialysis treatment if it appeared they might miss their Wheel-Trans. A nurse who expressed belief in the importance of exercise for patients commented that exercise is a low priority when "you have patients screaming, '...I want to get off [my dialysis], my Wheel-Trans is coming." 
Time

Lack of time was another barrier to exercise participation identified, particularly for exercise outside of dialysis sessions. As one patient explained:

You don't have time at home. I come to dialysis 3 days a week. I get up at 5am and I get home about 1:30-2pm and then I have to have a nap, I have household chores, I have bills, I have garbage, I have all kinds of stuff. I don't have time to exercise at home.

These comments were echoed by other participants who spoke of the time consuming nature of dialysis treatment and the difficulty of making time for exercise amidst daily responsibilities.

Lack of time proved to be a barrier in yet another way. In discussions about the nature of dialysis care, nurses identified lack of time as a barrier to encouraging patients to exercise. As one nurse commented:

You are literally going from one task to the next. So exercise, I certainly believe in exercise, for myself and for the patients. But it's a low priority when you have a patient crashing or someone is bleeding.

The low priority accorded to exercise encouragement when evaluated against competing patient care needs was expressed by many nurse participants. As a nurse explained,

That falls down very low on the priority list. I don't have one patient to keep alive, I have four and they are all very elderly and all very needy and sick so that tends to fall, if I have time that's like icing on the cake. I would love to do it if I had time.

\section{Equipment}

In both hospitals, only upright exercise bicycles were available for patients who wished to exercise during dialysis. Patients who were restricted to a reclining position during treatment were consequently unable to use the bicycles. As one nurse stated, "The ones with low blood pressure, they cannot do the bicycle because they have to be sitting up to do the bicycle, and with low blood pressure you have to lie them down with the legs higher." In addition, family care providers and patients expressed fears that using the bicycle would damage the fistula or chest 
line. In describing the exercise activities of her mother who receives dialysis, a family care provider explained,

Problem was that her line, she has a chest line, doesn't always work well with movement. But she is very motivated now...And she does want to begin exercising... but because of the line, I mean it's still kind of a fearful thing because you have this thing protruding out of your chest. So she is a little worried about what exercises would be safe, what wouldn't be safe.

Nurses consistently indicated the "risks" that the weight and size of the bicycles posed in the provision of dialysis care, including the hindering of emergency response. As one nurse indicated,

You cannot even get to the machine in case of an emergency. With the bike in the way it is very hard to get to the machines without jumping over things, it's really hard. I mean, God help you if a patient runs into some trouble during dialysis.

Nurses also repeatedly mentioned the potential for them to incur back injuries as a consequence of the weight of the equipment. As one nurse explained, "They're big, heavy and it's hard to move them. It hurts your back." Because of these concerns some nurses would rather not encourage the use of the bike by patients. As one nurse indicated,

Most of us are women, we're not big strong men, and it's hard for us to move these big things, and should I risk killing my back so the patient can exercise for ten minutes? So I think if we had user friendly equipment that was easier for us to move around that would make a difference.

Nurses even go as far as to discourage its use because of the potential risks to themselves. This is aptly captured in the following statement of a nurse: "I will discourage the patient because I don't want to hurt my back."

\section{Facilitators}

Sources of Motivation: Desire and Actual Improvement

Despite health-related impairment, many patients expressed a desire to exercise as exemplified in the following statement: "I enjoy exercising. I was always active. So the desire is 
still there and that's what pulls me through the days that are a little tougher because I'm not so well." For many patients, actual improvement from exercise gives them the motivation to adhere to exercise activities. As one patient described,

Keeps the blood pressure down, that's the big thing. That's why I exercise. And that's what motivates me, otherwise I have to take the blood pressure pill everyday, but now my blood pressure seems to be holding up alright.

Patients also spoke of psychological improvements they experienced from exercise. This is captured in the following statement of a patient: "It does help. It helps you mentally. You feel better because you have exercised, you feel tired physically but mentally and emotionally I think the exercise helps."

\section{Formal Incorporation of Exercise into the Treatment Plan}

When asked what would be required to facilitate patient engagement in exercise, staff overwhelmingly pointed to the need for change in the medical culture where no priority is accorded to exercise for wellness in the treatment plans for older hemodialysis patients.

Rehabilitation is prescribed for acute conditions but the absence of physicians' recommendation that patients participate in regular exercise as part of their health promotion poses a barrier to patients' participation in, and nurses' encouragement of exercise. As one patient stated:

I think you need to have at the outset, when you're first told that you have kidney disease, I think there needs to be a whole education program including nutrition, exercise, what's going to happen to you, and all the options that are open to you. Exercise is never even discussed but if it were discussed that would certainly motivate me.

Nurses similarly emphasized the need for formal incorporation of exercise into treatment plans, as exemplified in the following statement:

Exercise can be introduced. They already comply with the diet and fluid restrictions because they have to. So exercise should also be something they have to do. This has to be part of their education and once they get involved in their own treatment, in the same way that they 
are already with diet and fluid, I believe they will get the motivation and they will continue. And if it were part of their treatment, it would be part of what we do too.

These quotations emphasize how the exclusive focus of hemodialysis treatment on dietary and pharmacological management of patients meant that exercise for wellness, and the resources required for the support of such exercise, were not accorded priority by the health care team. As one nurse powerfully stated:

You can measure their blood pressure, you can see that their medications are working or not working, you can check their hemoglobin to see if they are anemic, but exercise is just a qualitative thing whether you feel good or not and I don't think that is a high priority.

\section{DISCUSSION}

Numerous studies have demonstrated that most dialysis patients are sedentary $[6,11]$. Patient disinterest [23] and lack of motivation [13] have been identified as the primary factors that impede exercise practices among dialysis patients. Contrary to these findings, data from this study indicate that patients desire exercise, indicating that more attention should be given to redressing barriers that extend beyond the patient.

Based on data showing that low physical functioning is directly related to poor outcomes in dialysis patients [24], guidelines recommend that dialysis patients be counseled and regularly encouraged by nephrology staff to exercise [25]. Despite such recommendations, the findings of this study indicate that staff rarely encouraged patients to exercise. These findings are consistent with the findings reported by Painter et al. [15] that of 100 dialysis staff surveyed, $34 \%$ never asked about physical limitations, $24 \%$ never or rarely encouraged patients to exercise, and only $32 \%$ regularly encouraged patients to exercise. The authors suggest that the reason for these findings is that in dialysis clinics, exercise is not part of the routine patient care plan, there are no programs in place within the units for exercise training, staff are not trained to encourage patients 
to exercise, nor are there mechanisms in place to support staff in helping patients to exercise [15].

The current study identifies three important barriers to patient exercise during dialysis that are amenable to change. First, the exercise equipment itself can pose a barrier. The exclusive use of upright exercise bicycles prevents the utilization by patients who are restricted to a reclining position during treatment, and their size and weight pose "risks" to the provision of dialysis care (the hindering of emergency response) and to the injury of staff in moving them (back injury) and can impede staff encouragement of their use.

Second, excessive patient care demands on nursing staff may leave them little or no time to engage in exercise promotion. This latter finding contradicts that of Painter et al. [15] who noted that most dialysis staff in their study had time in their daily routine to encourage patient exercise. This disparity may be accounted for by differences in staff ratios between US and Canadian dialysis clinics that allow American nurses more time to encourage exercise. Even within Canada there are differences in the number of patients per hemodialysis station, with Toronto facing the most critical shortage of resources [26].

Third, the punitive and restrictive policies of the publicly funded transportation service in Toronto strongly discouraged patient participation in exercise during dialysis. Because dialysis patients often have to agree to pick up times that may be hours in advance of their treatment, this contributes to their fatigue, which may act as a disincentive to exercise. In addition, in this study, as in other studies, transportation difficulties were associated with patient requests for shortened treatments [27]. Exercise is usually carried out in the early part of the dialysis session, and thus objectively there should be sufficient time for patients to exercise during dialysis and meet their scheduled pick-up time. However, because meeting the Wheel-Trans schedule is of such priority 
for patients, they are reluctant to engage in exercise because they perceive it as a potential threat to completing their treatment in a timely fashion.

Given that many of these barriers relate to aspects of the dialysis setting, home exercise on nondialysis days may be a reasonable alternative. However, barriers related to exercise training at home, such as lack of motivation and time, have been identified in other studies [28, 29]. While encouragement and individualized counseling have been found to increase patient activity at home [6], home exercise should not be pursued in exclusion to efforts to exercise during dialysis because of specific advantages unique to the latter. As Painter et al. [15] point out, patients are in the dialysis clinic three times a week, which provides a "captive" audience for receiving encouragement and guidance regarding exercise from health practitioners. Other benefits to exercise during dialysis are that it provides a productive activity during treatment [28], it is convenient and does not require extra time, and it results in fewer patients stopping exercise $[30,31]$. These findings support the need to address the systemic factors that may impede exercise during dialysis.

Findings of the current study underscore the importance of changing the culture of ESRD treatment programs to include a wellness approach that incorporates exercise counseling and encouragement into dialysis care. Painter et al. [15] have similarly argued that a change is needed from providing dialysis treatment alone to attending to patients' overall functioning [15]. Ridley [32] too has argued for a wellness-focused approach involving the provision of an exercise program to all medically stable hemodialysis patients. The consequence of not routinely incorporating exercise counseling and encouragement into dialysis care is that it signals to patients and families that it is not an important part of treatment and therefore not expected either during dialysis or at home [15]. Broadening understanding of the factors influencing exercise 
participation will help to facilitate the development of interventions that have a greater likelihood of success [33]. These efforts in turn will help to optimize the quality of life, functioning, and outcomes in older adults requiring hemodialysis. 


\begin{tabular}{|c|c|}
\hline \multicolumn{2}{|l|}{ Patients $(n=18)$} \\
\hline Age $($ mean $\pm \mathrm{SD})$ & $74.8 \pm 6.0$ \\
\hline Female & $6(33.3)$ \\
\hline \multicolumn{2}{|l|}{ Marital status, No. $(\%)^{*}$} \\
\hline Married & $11(78.6)$ \\
\hline Widow & $2(14.3)$ \\
\hline Single & $1(7.1)$ \\
\hline Time on hemodialysis (mean $\pm \mathrm{SD} * *$ ) & $4.8 \pm 2.5$ \\
\hline \multicolumn{2}{|l|}{ Staff $(n=24)$} \\
\hline Female & $22(91.7)$ \\
\hline Registered nurse & $24(100)$ \\
\hline Years of dialysis experience (mean $\left.\pm \mathrm{SD}^{* *}\right)$ & $13.7 \pm 5.7$ \\
\hline \multicolumn{2}{|l|}{ Family Caregivers $(n=7)$} \\
\hline Female & $4(57.1)$ \\
\hline \multicolumn{2}{|l|}{ Relation to patient, No. (\%) } \\
\hline Daughter & $2(28.6)$ \\
\hline Son & $2(28.6)$ \\
\hline Wife & $2(28.6)$ \\
\hline Husband & $1(14.2)$ \\
\hline $\begin{array}{l}\text { Missing data: } \\
* \mathrm{n}=4 \\
* * \mathrm{n}=1\end{array}$ & \\
\hline
\end{tabular}




\section{ACKNOWLEDGEMENTS}

This research was jointly funded by the Canadian Institutes of Health Research, Institute of Aging Pilot Grant Program, and the Collaborative Research Program: Rehabilitation \& LongTerm Care and the Canadian Nurses Foundation Nursing Care Partnership. Toronto Rehabilitation Institute receives funding under the Provincial Rehabilitation Research Program from the Ministry of Health and Long-Term Care in Ontario. We wish to thank all of the patients, family members and staff who participated in this study. We also gratefully acknowledge the administrative support we received from both hospitals that facilitated the implementation of the study. 


\section{REFERENCES}

1. Colangelo RM, Stillman MJ, Kessler-Fogil D, Kessler-Hartnett D. The role of exercise in rehabilitation patients with end-stage renal disease. Rehabil Nurs 1997; 22(6): 288-292.

2. Kouidi EJ. Central and peripheral adaptations to physical training in patients with end-stage renal disease. Sports Medicine 2001; 31(9): 651-655.

3. Pianta TF, Kutner NG. Improving physical functioning in the elderly dialysis patient: Relevance of physical therapy. ANNA J 1999; 26(1): 11-21.

4. Lysaght MJ. Maintenance dialysis population dynamics: Current trends and long-term implications. J Am Soc Nephrol 2002; 13: S37-S40.

5. Sioson ER, Kerfoot S, Ziat LM. Rehabilitation outcome of older patients with end stage renal disease and lower extremity amputation. J Am Geriatr Soc 1993; 41: 667-668.

6. Painter P, Carlson L, Carey S, Paul SM, Myll J. Physical functioning and health-related quality-of-life changes with exercise training in hemodialysis patients. Am J Kidney Dis 2000; 35(3): 482-492.

7. Painter P, Carlson L, Carey S, Paul SM, Myll J. Low-functioning hemodialysis patients improve with exercise training. Am J Kidney Dis 2000; 36: 600-608.

8. Mercer T, Crawford C, Gleeson NP, Naish PF. Low-volume exercise rehabilitation improves functional capacity and self-reported functional status of dialysis patients. Am J Phys Med Rehabil 2002; 81(3): 162-167.

9. Kong CH, Tattersall JE, Greenwood RN, Farrington K. The effect of exercise during haemodialysis on solute removal. Nephrol Dial Transplant 1999; 14: 2927-2931.

10. Stenvinkel P, Elinder C, Bárány P. Physical activity promotes health also among dialysis patients. Int J Cardiol 2000; 72: 299-300. 
11. Johansen KL, Kaysen G, Young B, Hung A, da Silva M. Longitudinal study of nutritional status, body composition, and physical function in hemodialysis patients. Am J Clin Nutr 2002; 77(4): 842-846.

12. Kouidi E, Grekas D, Deligiannis A, Tourkantonis A. Outcomes of long-term exercise training in dialysis patients: Comparison of two training programs. Clin Nephrol 2004; 61: S31-S38.

13. Goodman ED, Ballou MB. Perceived barriers and motivators to exercise in hemodialysis patients. Nephrol Nurs J 2004; 31(1): 23-29.

14. Johansen KL, Sakkas GK, Doyle J, Shubert T, Dudley RA. Exercise counseling practices among nephrologists caring for patients on dialysis. Am J Kidney Dis 2003; 41(1): 171178.

15. Painter P, Carlson L, Carey S, Myll J, Paul S. Determinants of exercise encouragement practices in hemodialysis staff. Nephrol Nurs J 2004; 31(1): 67-74.

16. Mays N, Pope C. Qualitative research: Rigour and qualitative research. BMJ 1995; 311: 109112.

17. Rowan M, Huston P. Qualitative research articles: Information for authors and peer reviewers. CMAJ 1997; 157: 1442-1446.

18. Morse J. Designing funded qualitative research. In: Denzin NK, Lincoln YS, editors. Handbook of Qualitative Research. Thousand Oaks, Calif.: SAGE, 1995: 220-235.

19. Denzin NK, Lincoln YS. Collecting and interpreting qualitative materials. Thousand Oaks, Calif.: SAGE, 1998.

20. Glesne C, Peshkin A. Becoming qualitative researchers: An introduction. White Plains, NY: Longman, 1992. 
21. Kuckartz U. Case-oriented quantification. In: Kelle U, editor. Computer-aided qualitative data analysis. London: Sage, 1995: 158-166.

22. Barbour RS. Checklists for improving rigour in qualitative research: A case of the tail wagging the dog? BMJ 2001; 322: 1115-1117.

23. Naglie G, Jassal V, Tomlinson G, Richardson R. Frailty in elderly hemodialysis patients at a large university hospital dialysis center. Gerontol 2002; 42(Special Issue 1): 239-240.

24. Curtin RB, Lowrie EG, DeOreo PB. Self-reported functional status: An important predictor of health outcomes among end-stage renal disease patients. Adv Ren Replace Ther 1999; 6(2): 133-139.

25. K/DOQI Workgroup. K/DOQI clinical practice guidelines for cardiovascular disease in dialysis patients. Am J Kidney Dis 2005; 45(4 Suppl 3): S1-153.

26. Mendelssohn DC, Chery A. Dialysis utilisation in the Toronto region from 1981 to 1992. Can Med Assoc J 1994; 150(7): 1099-1105.

27. Rocco M, Burkart J. Prevalence of missed treatments and early sign-offs in hemodialysis patients. J Am Soc Nephrol 1993; 4(5): 1178-1183.

28. Painter PL, Nelson-Worel JN, Hill MM, Thornbery DR, Shelp WR, Harrington AR, et al. Effects of exercise training during hemodialysis. Nephron 1986; 43(2): 87-97.

29. Williams A, Stephens R, McKnight T, Dodd S. Factors affecting adherence of end-stage renal disease patients to an exercise programme. BJSM 1991; 25(2): 90-93.

30. Konstantinidou E, Koukouvou G, Kouidi E, Deligiannis A, Tourkantonis A. Exercise renal rehabilitation: Comparison of three exercise programs. Journal of Rehabilitation Medicine 2001; 34: 40-45. 
31. Moug SJ, Grant S, Creed G, Boulton JM. Exercise during haemodialysis: Est of Scotland pilot study. Scottish Medical Journal 2004; 49: 14-17.

32. Ridley J, Hoey K, Ballagh-Howes N. The exercise-during -hemodialysis program: Report on a pilot study. CANNT 1999; 9: 20-26.

33. Rimmer JH, Riley B, Wang E, Rauworth A, Jurkowski J. Physical activity participation among persons with disabilities. Am J Prev Med 2004; 26(5): 419-425. 


\section{Appendix: Focus Group Guides}

\section{Patient Focus Group Guide}

1. What kinds of activities come to mind when you think of exercise?

2. Do you engage in any of these activities? If so, what is your experience (type of activity, frequency, group or individual activity, location)?

3. What led you to get involved in any of these activities (how did you find out about them, what do you understand the benefits of physical activity to be, what interests you about them)?

4. Do you receive any support (encouragement, instruction, praise) for doing these activities from other patients, family members, friends, or hospital staff (doctors, nurses, physiotherapists, etc.)?

5. Can you describe positive experiences you have had engaging in these activities?

6. What about negative experiences - can you describe negative experiences you have had engaging in these activities?

7. What health or other benefits have you gained from engaging in these activities?

8. For those of you who do not engage in exercise, can you explain the things that prevent you from doing so? (actual and anticipated/perceived consequences)?

9. What are some activities you wish you could do and why, and what would make it possible for you to do them?

\section{Staff Focus Group Guide}

1. When you think of exercise and older hemodialysis patients, what comes to mind? (types of exercises or activities; on the dialysis unit or off).

2. In what ways do you think that exercise has a health benefit to older dialysis patients? (have you seen evidence of this first hand?; when you think about your case load, can you provide a concrete patient example?)

3. In what ways do you think that exercise poses a health risk to older dialysis patients? (have you ever witnessed a negative event on the unit - if so, what happened?; are there patients that you think should not participate in any kind of exercise, and if so why?) 4. What is your unit's current approach to exercise? (do you have a formal exercise program?; what are the equipment and staffing conditions?)

5. How does your current nursing role fit with encouraging or discouraging exercise? (in what ways do you discuss activity/exercise habits with your patients?; in what ways do you encourage and/or discourage them to engage in exercise during dialysis sessions, on treatment days outside of dialysis, on non-treatment days at home?)

6. What are the facilitators and challenges to incorporating exercise into patients' treatment sessions? (structural barriers; medical barriers; and psychological or cultural barriers)

7. How does your patients' illness experience (physical and emotional) influence their involvement in exercise?

8. What other challenges do your patients face in doing exercise? 


\section{Family Care Provider Guide}

1. What kinds of activities come to mind when you think of exercise?

2. Does your relative engage in any of these activities? If so, what is his/her experience (type of activity, frequency, group or individual activity, location)?

3. What led your relative to get involved in any of these activities (how did he/she find out about them, what does he/she understand the benefits of physical activity to be, what interests him/her about them)?

4. Does he/she receive any support (encouragement, instruction, praise) for doing these activities from other patients, yourself or other family members, friends, or hospital staff (doctors, nurses, physiotherapists, etc.)?

5. Can you describe positive experiences he/she has had engaging in these activities?

6. What about negative experiences - can you describe negative experiences he/she has had engaging in these activities?

7. What health or other benefits has he/she gained from engaging in these activities?

8. If your relative does not engage in these activity, can you explain the things that prevent him/her from doing so? (actual vs. anticipated or perceived consequences)? 9. What are some activities your relative wishes he/she could do and why, and what would make it possible for him/her to do them? 